\title{
Safety and Efficacy of Insulin Detemir Among Adult Filipino Patients with Type 2 Diabetes: The Philippine Cohort of the Global $A_{1}$ chieve Study
}

\author{
Rosa Allyn Sy, ${ }^{1}$ Roberto Mirasol, ${ }^{2}$ Francis Pasaporte, ${ }^{3}$ Richard Elwyn Fernando, ${ }^{2}$ Cecilia Jimeno ${ }^{4}$ \\ ${ }^{1}$ Department of Medicine, Cardinal Santos Medical Center, Manila, Philippines \\ 2Department of Medicine, St. Luke's Medical Center, Quezon City, Philippines \\ ${ }^{3}$ Center for Diabetes Care, Iloilo, Visayas, Philippines \\ ${ }^{4}$ Department of Medicine, University of the Philippines-Philippine General Hospital, Manila, Philippines
}

\begin{abstract}
Objective. To present the results from the Philippine cohort of the $A_{1}$ chieve study receiving insulin detemir (IDet) \pm oral antidiabetic drugs.

Methodology. $A_{1}$ chieve is a multinational, 6-month, observational study of 66,726 people with type 2 diabetes mellitus (T2DM), both insulin users and non-insulin users, started on IDet, insulin aspart or biphasic insulin aspart in 28 countries across four continents. This subgroup analysis evaluates the safety and effectiveness of IDet in 988 subjects from the Philippines.

Results. At baseline the mean age, duration of diabetes and mean BMI were found to be $57 \pm 11.9 \mathrm{yrs}, 6.9 \pm 5.3 \mathrm{yrs}$ and $26 \pm 4.8 \mathrm{~kg} / \mathrm{m}^{2}$ respectively. Majority of subjects were insulin-naive $(84.3 \%)$ and glycemic control was poor in all the groups at baseline. At the end of 24 weeks, there were significant improvements following IDet initiation with a $2.1 \%$ reduction in mean $\mathrm{HbA}_{1 c}$. The $\mathrm{HbA}_{1 c}$ target of $<7 \%$ was achieved by $46.8 \%$ subjects at the study end. Although not statistically significant, there was a modest decrease in body weight in all the groups. There was no increase in the incidence of hypoglycemia among baseline insulin-naive subjects, while a significant reduction in hypoglycemia was seen among prior insulin users.
\end{abstract}

Conclusions. IDet appears to be an effective and safe option for individuals with T2DM in the Philippines. HbA $1 \mathrm{C}$ was lowered to target, and there was no increase in incidence of hypoglycemia and body weight with IDet.

Keywords: Type 2 diabetes mellitus, Insulin detemir, Philippines

\section{INTRODUCTION}

Worldwide, the prevalence of diabetes has reached epidemic proportions and the disease has become a major cause of morbidity and mortality. The global number of diabetics has been projected to rise approximately from 170 million in 2000 to 370 million in $2030 .{ }^{1}$ In June 2000, diabetes mellitus (DM) was declared a major and growing health problem in the Western Pacific Region, including the Philippines. ${ }^{2}$ According to two Philippine surveys, National Nutrition and Health Survey I (NNHeS I) and NNHeS II, conducted in 2003 and 2008, respectively, the overall prevalence of diabetes based on FBG increased from $3.3 \%$ (2003) to $3.9 \%$ (2008), while the true prevalence of diabetes based on FBG and questionnaire responses, increased from $4.4 \%$ (2003) to $5.2 \%$ (2008). ${ }^{3}$ The International Diabetes Federation (IDF) reported that the prevalence of diabetes in adults in the 20-79 year age group in the Philippines was $6.5 \%$ and estimated it to be $7.9 \%$ by $2025 .{ }^{4}$ However, a recent study by Soria et al,

ISSN 0857-1074

Printed in the Philippines

Copyright $(0) 2013$ by the JAFES

Received December 5, 2012. Accepted April 23, 2013.

The educational grant for the study was provided by Novo Nordisk International Operations, Zurich, Switzerland. carried out in a cohort of 1749 individuals has reported much higher 9-year (1999-2007) incidence and prevalence rates of type $2 \mathrm{DM}$ at $16.3 \%$ and $28.0 \%$ respectively. ${ }^{1}$ This signifies a major healthcare burden for the productive population of a developing country like the Philippines in terms of the high cost of antidiabetic drugs, diagnostics and other treatment modalities.

Insulin is frequently reserved for an advanced stage of the disease and often prescribed after multiple oral antidiabetic drugs fail. Although insulin is the most effective treatment for insulin deficient type 2 diabetes with proven efficacy in lowering $\mathrm{HbA}_{1 \mathrm{c}}$, there is strong resistance to introduce insulin at an earlier stage of the disease. ${ }^{5}$ In the Diabetes Attitudes, Wishes, and Needs (DAWN) study, patients rated the clinical efficacy of insulin as low and started blaming themselves if they had to start insulin therapy. Fifty to fifty-five percent of nurses and general practitioners delayed insulin therapy until absolutely necessary, but delay was significantly less 
among specialists and opinion leaders. Delay of insulin therapy was significantly less likely when physicians and nurses see their patients as more adherent to medication or appointment regimens. ${ }^{6}$

Evidence based guidelines from the IDF also recommend the use of insulin even in early stages of the disease if target $\mathrm{HbA}_{1 c}$ of $\leq 7.5 \%$ is not achievable by lifestyle modification and two or three oral antidiabetic drugs (OADs). ${ }^{7}$ Furthermore, the recent position statement of the American Diabetes Association (ADA) and the European Association for the Study of Diabetes (EASD) recommend the use of basal insulin along with metformin in patients with high baseline level of $\mathrm{HbA}_{1 \mathrm{c}}(\geq 9.0 \%) .{ }^{8}$ However, such recommendations are not rigorously followed, possibly due to the physician's perceived complexity of the therapy and concerns about its adverse effects, namely hypoglycemia and weight gain.7,9 To address such concerns, modern basal insulin analogues such as insulin detemir (IDet) have been developed to achieve better blood glucose control with relatively lower risks of adverse events. ${ }^{5}$

IDet is a modified form of insulin which has a deletion of the terminal B chain amino acid (B30), and a fatty acid side chain (myristic acid) attached at B29 position. These changes facilitate dihexameric complexes to form in the injection depot and also enable binding with albumin. 5,10 Therefore, IDet provides sustained control of blood glucose levels at night-time and between meals and its pharmacological profile matches with that of endogenous basal insulin secretion. ${ }^{5,10,11}$ It controls blood glucose effectively and considerably reduces the risk of hypoglycemia by providing a steady release of insulin in diabetic patients. ${ }^{10,11}$ Sustained effect over 24 hours also facilitates once daily use and enhances patient convenience and compliance with therapy. ${ }^{10}$ Many randomised controlled trials (RCTs) have shown that the use of IDet significantly reduces $\mathrm{HbA}_{1 c}$ and blood glucose levels and is associated with a very low risk of hypoglycemia. ${ }^{5,10}$ However, the beneficial effects reported in RCTs require application and validation in routine clinical practice amongst different patient populations, in varying stages of the disease, with co-morbidities and on multiple medications. It is generally accepted that such complex information can be derived from large observational studies. ${ }^{12}$ Data generated from observational studies can complement those from RCTs by providing an insight into how treatments perform in day to- day practice in more clinically representative patient populations. ${ }^{13}$ The Aichieve study is one of the largest observational studies on insulin therapy to date. It was designed to assess the real life safety and effectiveness of insulin analogues in large diabetic populations (more than 65,000 patients comprising of insulin-naive patients and prior insulin users from 28 countries). The study also evaluated the effect of insulin analogues on the health related quality of life (HRQoL) scores of the patients. ${ }^{7}$
The safety and effectiveness of IDet in the Philippine population is not well established, as there is scarce published data exploring the use of this analogue in routine clinical practice. Therefore, the objective of the present study is to analyse the safety, effectiveness and HRQoL parameters of the Archieve study participants in the Philippine cohort, treated with IDet.

\section{METHODOLOGY}

\subsection{Study overview}

The Archieve study is a 24-week, international, prospective, multicentre, non-randomised, observational study of people with T2DM who had begun using basal IDet (Levemir, ${ }^{\circledR}$ Novo Nordisk, Denmark), bolus insulin aspart (NovoRapid, ${ }^{\circledR}$ Novo Nordisk) and biphasic insulin aspart 30 (NovoMix 30, ${ }^{\circledR}$ Novo Nordisk), alone or in combination with OADs. It was undertaken to evaluate their clinical safety and effectiveness in routine clinical use outside the Western economies. ${ }^{7}$ The study was carried out in 28 countries across Asia, Africa, Latin America and Europe.

\subsection{Study eligibility}

The study was conducted in accordance with the principles of Declaration of Helsinki and good clinical practice guidelines. Ethics committee approval before study commencement, and signed informed consent from all participants prior to participation in the study was obtained. Patients of type 2 diabetes on any current and prior medications other than the insulin analogue being evaluated were included. Patients, who had been started on the study insulin within 4 weeks prior to the study start, were also considered eligible for participation. People with hypersensitivity to the study drug or excipients were excluded. Women who were pregnant, breast-feeding or had planned for pregnancy within 6 months of the study were excluded. Participants were free to withdraw at will at any time during the study. If they withdrew, the data collected were used for analysis until the point when consent was withdrawn.

\subsection{Study objectives and assessment}

The primary objective was to evaluate safety of the insulin analogues by the incidence of serious adverse drug reactions (SADRs), including major hypoglycemic events recorded from baseline to final visit. Minor hypoglycemic episodes and nocturnal hypoglycemia were also assessed. Major hypoglycemic events were defined as events with severe central nervous system symptoms, consistent with hypoglycemia, for which the person was unable to selftreat, and accompanied by plasma glucose $<3.1 \mathrm{mmol} / \mathrm{L}$ or $56 \mathrm{mg} / \mathrm{dL}$, or reversal of symptoms after either food intake or glucagon or intravenous glucose administration. Minor hypoglycemia was any event, with or without symptoms of hypoglycemia, with a plasma glucose reading below 3.1 
$\mathrm{mmol} / \mathrm{L}$ or $56 \mathrm{mg} / \mathrm{dL}$ and the participant was able to selftreat. Nocturnal hypoglycemia was defined as a symptomatic event consistent with hypoglycemia that occurred during sleep between bedtime after the evening insulin injection and before getting up in the morning.

Effectiveness of therapy was determined from the change in $\mathrm{HbA}_{1 c}$, fasting plasma glucose (FPG), postprandial plasma glucose (PPPG) and body weight between baseline and interim and final visits, and change in systolic blood pressure (SBP) and lipid profile at final visit. In addition, the effect of insulin analogue therapies on HRQoL of the participants was also evaluated.

The insulin therapies were prescribed by the physician during routine clinical practice, were commercially available, and were funded according to local practice in normal routine care. Starting dose of insulin detemir was based on physician's discretion.

There were no defined study-related procedures and measurements aside from those made by the treating physician team only as required during routine clinical care. Data points were captured at baseline, interim (around 12 weeks from baseline) and final (around 24 weeks from baseline) visit. The time period of 4 weeks prior to the baseline visit, was defined as a pre-study period. Data was collected from the physicians' clinical notes, and participants' recall and self-monitoring diary/meter at each visit and transferred to a standard case report form (CRF).

HRQoL was measured using the EQ-5D questionnaire and EQ visual analogue scale (EQ VAS) at baseline and after 24 weeks of therapy with insulin analogues. EQ VAS included a rating for an individual's current state, measured by a standard vertical $20 \mathrm{~cm}$ scale with score ranging from 0 (worst imaginable health) to 100 (best imaginable health). EQ-5D questionnaire consisted of five dimensions (mobility, self-care, usual activities, pain/discomfort, anxiety/depression) which was scored as 1,2 or 3 depending on the level of severity. These different dimensions were converted to a single utility value, anchored by ' 1.00 ' representing full health and ' 0.00 ' representing the state 'dead.'

Participants were recruited between January 2009 and June 2010 and a total of 66,726 people were included in the study. ${ }^{9}$ In this paper, we present the results of analysis conducted on the data of participants from the Philippines, treated with IDet (Levemir, Novo Nordisk, Denmark).

\section{Statistical methods}

The sample size calculation for the entire global cohort was based on the number of patients $(60,000)$ exposed for 6 months required to confirm a frequency of $\geq 15$ events/100,000 patient-years of any one SADR, including major hypoglycemic events, at the $95 \%$ confidence level. Statistical analyses were performed for the entire cohort and for the entire cohort classified as insulin-naive or prior insulin users. Descriptive statistics were used to summarise continuous variables and frequency tables (number and percentage) were used for discrete variables. All statistical analyses were two-sided, with 5\% significance level, unless otherwise stated. For the change in hypoglycemia from baseline, the percentage of patients reporting at least one event was analysed using Fisher's exact test. The change from baseline in $\mathrm{HbA}_{1 c}, \mathrm{FPG}, \mathrm{PPPG}$, SBP, body weight, blood lipids and HRQoL was analysed using a paired t-test using baseline and end-of-study values. Data analyses were performed by Novo Nordisk using SAS (Version 9.1.3).

\section{RESULTS}

\section{Baseline characteristics}

A summary of patient demographics at baseline is presented in Table 1 . Of the total 988 patients on IDet included in the subgroup analysis of Philippines cohort, 833 patients were insulin therapy naive $(84.3 \%)$ and 155 patients $(15.7 \%)$ were on prior insulin therapy. Female participants constituted $54.6 \%$ of the entire cohort. The participants were $56.6 \pm 11.6$ years and $59.4 \pm 13.2$ years of age in insulin-naive and prior insulin user groups respectively. Majority of the patients already had established diabetes during the time of enrolment and the duration of diabetes in the insulin therapy naive group was $6.5 \pm 4.9$ years while it was $8.7 \pm 6.5$ years in those on prior insulin therapy. Body mass index (BMI) was comparable in both the groups (26.1 vs $\left.25.9 \mathrm{~kg} / \mathrm{m}^{2}\right)$. The baseline $\mathrm{HbA}_{1 \mathrm{c}}$ levels were also similar in insulin-naive patients at $9.3 \pm 1.9 \%$ and prior insulin users at $9.0 \pm 1.8 \%$. The PPPG (13.5 vs $13.7 \mathrm{mmol} / \mathrm{l}$ ) and FPG (11.5 vs 10.6 $\mathrm{mmol} / \mathrm{l}$ ) values were also comparable (Table 1 and Table 2).

Table 1. Baseline characteristics of the Philippines cohort, $\mathrm{N}=988$

\begin{tabular}{lccc}
\hline & $\begin{array}{c}\text { Entire } \\
\text { cohort }\end{array}$ & Insulin-naive & $\begin{array}{c}\text { Prior insulin } \\
\text { users }\end{array}$ \\
\hline $\mathrm{N}(\%)$ & 988 & $833(84.3)$ & $155(15.7)$ \\
Sex, M/F † (\%) & $450 / 537$ & $377 / 455$ & $73 / 82$ \\
& $(45.4 / 54.6)$ & $(45.3 / 54.7)$ & $(47.1 / 52.9)$ \\
Age (yrs) & $57.00(11.9)$ & $56.6(11.6)$ & $59.4(13.2)$ \\
Duration of & $6.9(5.3)$ & $6.5(4.9)$ & $8.7(6.5)$ \\
diabetes (yrs) & & & \\
Bodyweight (kgs) & $66.5(14.1)$ & $66.7(13.9)$ & $65.9(15.3)$ \\
BMl § (kg/m2) & $26.0(4.8)$ & $26.1(4.8)$ & $25.9(5.1)$ \\
HbA1c (\%) & $9.3(1.9)$ & $9.3(1.9)$ & $9.0(1.8)$ \\
\hline †- male/female; §- body mass index; Data expressed in Mean \\
(Standard deviation) for all variables except N and Sex \\
Source:
\end{tabular}

\section{Safety and Effectiveness}

\section{1. Glycemic control and insulin dose}

Treatment with IDet led to significant improvement in glycemic control over the 24 week period. In the entire cohort, the mean reduction in $\mathrm{HbA}_{1 c}$ from baseline was 2.1 
Table 2. The effectiveness of IDet using various parameters of glycemic control

\begin{tabular}{|c|c|c|c|c|c|}
\hline & & Baseline & 24 weeks & Mean difference between & \\
\hline & & $\mathrm{X}, \mathrm{SD}$ & $\mathrm{X}, \mathrm{SD}$ & baseline $\& 24$ weeks & $p$-value \\
\hline \multirow{3}{*}{$\mathrm{HbA} 1 \mathrm{c}(\%)$} & Entire cohort $(\mathrm{N}=460)$ & $9.3(1.9)$ & $7.1(1.1)$ & $-2.1(1.6)^{\star \star}$ & $<0.001$ \\
\hline & Insulin-Naive at baseline $(\mathrm{N}=402)$ & $9.3(1.9)$ & $7.1(1.1)$ & $-2.2(1.7)^{\star *}$ & $<0.001$ \\
\hline & Prior insulin users $(\mathrm{N}=155)$ & $9.0(1.8)$ & $7.1(1.2)$ & $-1.9(1.5)^{\star *}$ & $<0.001$ \\
\hline Proportion with $\mathrm{HbA} 1 \mathrm{c}$ & Entire cohort $(\mathrm{N}=460)$ & 6.6 & 46.8 & - & - \\
\hline \multirow[t]{2}{*}{$<7 \%$} & Insulin-Naive at baseline $(\mathrm{N}=402)$ & 6.0 & 45.5 & - & - \\
\hline & Prior insulin users $(N=155)$ & 9.6 & 55.1 & - & - \\
\hline \multirow{3}{*}{$\begin{array}{l}\text { Fasting plasma glucose } \\
(\mathrm{mmol} / \mathrm{l})\end{array}$} & Entire cohort $(\mathrm{N}=579)$ & $11.4(4.1)$ & $6.7(2.1)$ & $-4.7(4.1)^{\star \star}$ & $<0.001$ \\
\hline & Insulin-Naive at baseline $(\mathrm{N}=487)$ & $11.5(4.1)$ & $6.6(2.1)$ & $-4.9(4.2)^{\star *}$ & $<0.001$ \\
\hline & Prior insulin users $(\mathrm{N}=92)$ & $10.6(4.1)$ & $6.9(2.1)$ & $-3.7(3.2)^{\star *}$ & $<0.001$ \\
\hline \multirow{3}{*}{$\begin{array}{l}\text { Post prandial plasma } \\
\text { glucose }(\mathrm{mmol} / \mathrm{l})\end{array}$} & Entire cohort $(\mathrm{N}=67)$ & $13.6(4.7)$ & $8.2(2.4)$ & $-5.4(4.4)^{* *}$ & $<0.001$ \\
\hline & Insulin-Naive at baseline $(\mathrm{N}=55)$ & $13.5(4.5)$ & $8.2(2.6)$ & $-5.3(4.1)^{\star \star}$ & $<0.001$ \\
\hline & Prior insulin users $(\mathrm{N}=12)$ & $13.7(6.1)$ & $7.9(1.6)$ & $-5.7(5.0)^{*}$ & $<0.01$ \\
\hline
\end{tabular}

$\%(\mathrm{p}<0.001)$, mean reduction in FPG and PPPG from baseline were $4.7 \mathrm{mmol} / \mathrm{L}(\mathrm{p}<0.001)$ and $5.4 \mathrm{mmol} / \mathrm{L}$ $(\mathrm{p}<0.001)$ respectively. Mean $\mathrm{HbA}_{1 c}$ was reduced by $2.2 \%$ and $1.9 \%$ respectively in insulin naive and prior insulin users. An analysis further into the treatment naive patients revealed a reduction of $2.5 \%$ in $\mathrm{HbA}_{1 c}$ from baseline $(9.5 \pm$ 1.8). Treatment naive were those insulin naive patients who had not received any anti-diabetic therapy, i.e., even OAD, prior to enrolment. The other parameters (FPG \& PPPG) also showed reductions of similar extent as of entire cohort, in the insulin-naive patients and prior insulin users. Target $\mathrm{HbA}_{1 c}$ level of $<7 \%$ was achieved by $46.8 \%$ of participants of the entire cohort after 24 weeks of IDet therapy. Target $\mathrm{HbA}_{1 \mathrm{c}}$ level of $<7 \%$ was reached by $45.5 \%$ of insulin-naive and $55.1 \%$ of prior insulin users following IDet treatment (Table 2).

During the study the dose of insulin in the insulin-naive group was up titrated to $24.6 \pm 14.1 \mathrm{U} /$ day by 24 weeks. In prior insulin users the pre-IDet dose was $28.6 \pm 16.4 \mathrm{U} /$ day, this changed to $21.9 \pm 10.8 \mathrm{U} /$ day at baseline and increased to $27.7 \pm 13.6 \mathrm{U} /$ day at the end of study. Furthermore, the pattern of OAD use in the patients also changed slightly during 24 weeks of the study. Use of metformin in the entire cohort was relatively stable through the study period, with $77.1 \%, 70.5 \%$ and $73.5 \%$ of the patients using the drug during pre-study period, at baseline and 24 weeks respectively. The use of thiazolidinediones also did not change significantly during the study. However, the use of sulfonylureas decreased from $71.1 \%$ during prestudy to $51.6 \%$ at baseline and to $45.2 \%$ at the end of the study period. Metformin and SU was also the most common regimen used by patients in combination throughout the study period (pre study: $32.0 \%$, baseline: $22.2 \%$, study end: $20.5 \%$ )

\subsection{Hypoglycemia}

At baseline, overall 123 hypoglycemic events (1.62 events/person year) were observed in 55 (5.6\%) individuals of the entire cohort. Treatment with IDet led to a statistically significant reduction in the rate of overall hypoglycemia in the entire cohort, with 36 (0.56 events/person year) events occurring in 20 patients $(\mathrm{p}<0.001)$. In prior insulin users, the reduction was remarkable as overall hypoglycemic events fell significantly from 4.36 events/person year at baseline to 0.52 events/person year occurring at 24 weeks. Although, there was also a reduction in the rate of hypoglycemia in insulin-naive patients from 1.11 events/person year to 0.57 events/person year, it was not statistically significant. Eleven major hypoglycemic episodes ( 0.14 events/person year) in 7 patients were reported from the entire cohort at baseline. The incidence of major hypoglycemia at baseline was higher in insulin-naive patients $(0.16$ events/person year) compared to prior insulin users (0.08 events/person year). After 24 weeks of IDet therapy there was no report of major hypoglycemic event occurring in any participant. Minor hypoglycemic events in the entire cohort decreased significantly from 1.47 events/person year to 0.56 events/person year following 24 week treatment with IDet $(\mathrm{p}<0.05)$. Nocturnal hypoglycemia was also significantly reduced following treatment with IDet and the reduction was more evident in prior insulin users where it fell from 1.59 events/person year to 0.1 events/ person year $(\mathrm{p}<0.001)$ (Table 3).

Table 3. Incidence of hypoglycemia and the effect of IDet on body weight, $\mathrm{N}=724$

\begin{tabular}{|c|c|c|c|c|}
\hline \multicolumn{2}{|c|}{$\begin{array}{l}\text { (Percent with event/event } \\
\text { per person-year) }\end{array}$} & \multirow{2}{*}{$\begin{array}{l}\begin{array}{l}\text { Entire } \\
\text { cohort }\end{array} \\
5.6 / 1.62\end{array}$} & \multirow{2}{*}{$\begin{array}{c}\begin{array}{c}\text { Insulin- } \\
\text { naive }\end{array} \\
3.8 / 1.11\end{array}$} & \multirow{2}{*}{$\begin{array}{c}\begin{array}{c}\text { Prior } \\
\text { insulin } \\
\text { users }\end{array} \\
14.8 / 4.36\end{array}$} \\
\hline Overall & Baseline & & & \\
\hline Hypoglycemia $^{\ddagger}$ & 24 weeks & $2.4 / 0.56^{\star \star}$ & $2.4 / 0.57$ & $2.4 / 0.52^{\star *}$ \\
\hline Minor & Baseline & $5.4 / 1.47$ & $3.7 / 0.95$ & $14.2 / 4.28$ \\
\hline Hypoglycemia $^{\ddagger}$ & 24 weeks & $2.4 / 0.56^{\star *}$ & $2.4 / 0.57$ & $2.4 / 0.52^{* *}$ \\
\hline Major & Baseline & $0.7 / 0.14$ & $0.7 / 0.16$ & $0.6 / 0.08$ \\
\hline Hypoglycemia ${ }^{\ddagger}$ & 24 weeks & $0.00 / 0.00^{*}$ & $0.00 / 0.00^{*}$ & $0.00 / 0.00$ \\
\hline Nocturnal & Baseline & $2.1 / 0.53$ & $1.3 / 0.33$ & $6.5 / 1.59$ \\
\hline \multirow[t]{2}{*}{ Hypoglycemia $^{\ddagger}$} & 24 weeks & $0.7 / 0.12^{*}$ & $0.7 / 0.13$ & $0.8 / 0.10^{*}$ \\
\hline & $\mathrm{N}$ & 724 & 604 & 120 \\
\hline Body weight & Baseline & $63.3(13.1)$ & $66.6(13.2)$ & $64.9(12.6)$ \\
\hline \multirow{2}{*}{ (kg) } & 24 weeks & $66.1(12.7)$ & $66.4(12.8)$ & 64.4 (12.3) \\
\hline & Change & $-0.2(4.8)$ & $-0.1(4.6)$ & $-0.5(5.6)$ \\
\hline
\end{tabular}

\subsection{Body weight, blood lipids and blood pressure}

Following IDet therapy for 24 weeks, there was no statistically or clinically meaningful change in body weight in either insulin-naive patients or prior insulin users. (Table 3) Total cholesterol level for the entire cohort decreased significantly from $5.7 \pm 1.3 \mathrm{mmol} / \mathrm{L}$ to $4.7 \pm 0.8$ $\mathrm{mmol} / \mathrm{L}$ after 24 weeks of therapy $(\mathrm{p}<0.001)$. Statistically 
significant reduction in level of LDL cholesterol from baseline value of $3.6 \pm 1.3 \mathrm{mmol} / \mathrm{L}$ to $2.8 \pm 0.9 \mathrm{mmol} / \mathrm{L}$ was noted in the entire cohort post treatment with IDet $(\mathrm{p}<$ 0.001 ). Serum triglyceride level decreased from $2.1 \pm 1.0$ $\mathrm{mmol} / \mathrm{L}$ at baseline to $1.5 \pm 0.6 \mathrm{mmol} / \mathrm{L}$ post treatment in the entire cohort $(p<0.001)$. However, there was no significant change in the HDL level and it remained stable during the study period. A reduction of $6 \mathrm{~mm} \mathrm{Hg}$ in the systolic blood pressure (SBP) of the entire cohort, from $129.2 \pm 18.6 \mathrm{~mm} \mathrm{Hg}$ at baseline to $123.2 \pm 13.9 \mathrm{~mm} \mathrm{Hg}$ at 24 weeks was observed $(p<0.001)$. The reduction in SBP after IDet therapy was clinically similar in insulin-naive patients and prior insulin users.

\section{Health-related quality of life}

At baseline, the EQ VAS scores for the entire cohort was 70.7 which was similar to that recorded from insulin-naive patients (70.9) and prior insulin users (69.8). The scores showed statistically significant improvement following IDet therapy and changed by +12.6 points for the entire cohort $(\mathrm{p}<0.001)[+12.3$ points for insulin-naive patients $(\mathrm{p}<0.001)$ and +14.8 points in prior insulin users $(\mathrm{p}<0.001)]$. Similarly across all the summary dimensions of EQ-5D, significant improvements were observed following IDet therapy. Notably, prior insulin users reported higher improvement of 0.116 points from baseline value of 0.754 in the EQ-5D score as compared to insulin-naive patients who reported a change of 0.097 points from baseline value of 0.816 after treatment with IDet.

\section{DISCUSSION}

The present subgroup analysis was carried out to assess the safety, effectiveness and change in HRQoL parameters in A1chieve study participants in Philippines treated with IDet. The extent of $\mathrm{HbA}_{1 c}$ reduction (2.1\%) observed with 6 month IDet therapy in Filipino patients was statistically significant and clinically relevant and, similar to that observed in the global analysis of the Archieve study, where the reduction in $\mathrm{HbAl}_{1}$ was reported to be $2.1 \%$ in insulin-naive and $1.6 \%$ in prior insulin users. ${ }^{9}$ Significant reductions in FPG and PPPG in insulin-naive and prior insulin users were also observed and were consistent with that reported from the global analysis of the A1chieve study. These reductions were significant in both insulinnaive and insulin experienced patients. The UK prospective diabetes study (UKPDS), had shown that every $1.0 \%$ reduction in $\mathrm{HbA}_{1 c}$ is associated with a $43 \%$ reduction in the risk of amputation or death from peripheral vascular disease, a $37 \%$ reduction in microvascular disease, and a $16 \%$ reduction in heart failure. ${ }^{7,14}$ Therefore, a $2 \%$ reduction in $\mathrm{HbA}_{1 c}$ levels with IDet would translate into obvious significant reductions in diabetic complications. Importantly, $45.5 \%$ of insulin-naive patients achieving target $\mathrm{HbA}_{1 \mathrm{c}}$ of $<7 \%$ suggests the benefit of early initiation of IDet in newly diagnosed cases. Although effectiveness of IDet in controlling the blood glucose of insulin-naive patients may have been expected, its effect in insulin experienced patients was not entirely anticipated. Prior insulin users who were suffering from diabetes for an average of $8.7 \pm 6.5$ years also responded well to IDet treatment, and $\mathrm{HbA}_{1 \mathrm{c}}$ and FPG levels were reduced by $1.9 \%$ and $3.7 \mathrm{mmol} / \mathrm{L}$ respectively. There was a marginal reduction in body weight accompanied by statistically significant reductions in total cholesterol, LDL cholesterol and triglycerides. It is possible that lowering of blood lipids could also have contributed partly towards better glycemic control observed in this study. ${ }^{15}$

It has been reported that diabetic patients in the Philippines often have poor glycemic control. In a multicenter, multi-country survey in which the Philippines participated, nearly $60 \%$ of Philippine participants showed $\mathrm{HbA}_{1 \mathrm{c}}$ levels of $>8 \%{ }^{4}$ However, the present study indicates that clinically meaningful glycemic control could be achieved with IDet in Filipino patients; which would be at par with other diabetic populations across the world receiving this agent. Our observations are similar to that observed in German cohort of the PREDICTIVE study. In that study, addition of once daily basal IDet in patients on oral antidiabetic drugs led to a significant decrease in $\mathrm{HbA}_{1 c}$ level by $1.29 \%$, as compared to $0.6 \%$ with NPH insulin plus oral antidiabetic drugs and $0.59 \%$ with insulin glargine plus oral antidiabetic drugs. ${ }^{16}$ Other studies have reported that the degree of glycemic control achievable with once daily IDet is similar to that with once daily insulin glargine albeit with a lower risk of weight gain. ${ }^{17,18}$ Interestingly, switching from glargine insulin based regimen to IDet had also led to further $0.6 \%$ reduction in $\mathrm{HbA}_{1 c}{ }^{19}$

Patients with long duration of diabetes often require intensive insulin therapy for optimal glycemic control. However, intensive insulin therapy whilst improving glycemic control is associated with increased risk of hypoglycemia leading to significant morbidity and mortality. ${ }^{20}$ Hypoglycemia may have particularly serious outcomes in elderly or frail people with type 2 diabetes. The incidence for severe hypoglycemia has been reported to be 11.8 and 0.9 events per 100 patient-years for patients with type 2 diabetes treated with insulin and sulfonylureas respectively. ${ }^{21}$ The UK Hypoglycemia Study Group examined the frequency of hypoglycemia prospectively for 9-12 months in type 2 diabetics who were being treated in secondary care. They reported the prevalence of severe hypoglycemia to be $25 \%$ in patients who were using insulin for more than 5 years. ${ }^{21,22}$ Episodes of severe hypoglycemia also increases hospital visits and consequently drives up treatment costs. In the current analysis, no major hypoglycemic event was observed over the 6 month period, even in prior insulin users.

In addition, the incidence of minor and nocturnal hypoglycemic events also decreased significantly. This is in agreement with the global analysis of Aichieve study data where the rate of major hypoglycemic episodes in 
prior insulin users decreased from 0.88 events/person-year at baseline to 0.01 events/person-year after 6 months therapy with IDet. Moreover, the use of sulfonylureas went down in the entire cohort from $51.6 \%$ at baseline to $45.2 \%$ at the end of the study, which may indicate better control with insulin regimen alone. Our observations regarding safety of IDet validate those reported from other studies. In the German cohort of PREDICTIVE study, major hypoglycemia was significantly reduced by $55 \%$ and $51 \%$ respectively in prior NPH and glargine insulin users who switched to IDet. ${ }^{19}$ The safety related findings of the present study are important as they have been obtained during routine clinical practice and are more realistic than those observed within the controlled environment of clinical trials. On the other hand there might also be under reporting of hypoglycemia in this study. The low incidence of adverse event therefore suggests that diabetic patients from the Philippines satisfactorily tolerate IDet therapy.

It was not surprising that patient reported HRQoL indices markedly improved by the end of the study. The EQ-5D instrument used in the study has been previously validated for use in diabetic patients. ${ }^{23}$ The significant increase observed in the EQ VAS score and EQ-5D score at 6 months was testament to the positive patient experience with IDet therapy. The improvement in these scales paralleled with the improvement in glycemic control, and is in conformity with the study of Shah et al, which reported similar improvement in HRQoL scores in participants of the Archieve study following the use of IDet. $^{24}$

Moreover, the exceptional results observed in glycemic control and hypoglycemia with insulin detemir even in prior insulin users, may need a clear explanation.

This subgroup analysis has provided novel insight into the safety and efficacy of IDet in Filipino, however, being an observational study, there are some inherent limitations. Concomitant medications were not controlled. The study was non-randomised and no standardised treatment protocol was enforced. It also lacks a control arm, and most safety parameters were based on participant recall or diaries. The data of insulin use in the pre-study period has not been analysed and will be communicated in future publications. The possible reasons for the results observed in glycemic control and hypoglycemia with insulin detemir may be due to greater therapeutic effect of detemir compared to other insulins (glargine and NPH). This was also observed in European cohort of PREDICTIVE study in T2DM patients, where switching to detemir from prior insulins (glargine and NPH) resulted in more pronounced effect, with $\mathrm{HbA} 1 \mathrm{c}$ reductions up to $0.6 \%{ }^{25}$ The reduced subject variability, improved diabetes care, and continued follow-up also resulted in a better compliance, thus achieving better glycemic control.

\section{CONCLUSIONS}

The overall results of this subgroup analysis suggest that the use of IDet in insulin-naive as well as insulin experienced Filipino patients led to a significant improvement in glycemic control. Moreover, such improved control of blood glucose was achieved with reduced risk of hypoglycemia and improvement in patient's HRQoL scores. These favorable effects were observed without any concomitant detrimental effect on body weight or lipid profile. Thus, the observations of this study while providing a practical insight on the utility of IDet may acquaint Philippine physicians to the utility of basal insulin therapy in patients of type 2 diabetes and enable them towards making critical treatment decisions for their patients.

\section{Acknowledgements}

We thank Novo Nordisk for providing an educational grant for the study and WorkSure (India) for providing medical writing assistance.

\section{References}

1. Soria ML, Sy RG, Vega BS et al. The incidence of type 2 diabetes mellitus in the Philippines: A 9-year cohort study. Diabetes Res Clin Pract. 2009; 86(2):130-3.

2. Baltazar JC, Ancheta CA, Aban IB, Fernando RE, Baquilod MM. Prevalence and correlates of diabetes mellitus and impaired glucose tolerance among adults in Luzon, Philippines. Diabetes Res Clin Pract. 2004; 64(2):107-15.

3. Sy RG, Morales DD, Dans AL et al. Prevalence of atherosclerosisrelated risk factors and diseases in the Philippines. J Epidemiol. 2012; 22(5):440-7.

4. Higuchi M. Access to diabetes care and medicines in the Philippines. Asia Pac J Public Health. 2010; 22(3 Suppl):96S-102S.

5. John M. Basal insulin analogues--A review of recent data on efficacy and safety. J Assoc Physicians India. 2011; 59 Suppl:25-8.

6. Peyrot M, Rubin RR, Lauritzen $\mathrm{T}$ et al. Resistance to insulin therapy among patients and providers: results of the cross-national Diabetes Attitudes, Wishes, and Needs (DAWN) study. Diabetes Care. 2005; 28(11):2673-9.

7. Shah SN, Litwak L, Haddad J, Chakkarwar PN, Hajjaji I. The Aichieve study: A 60 000-person, global, prospective, observational study of basal, meal-time, and biphasic insulin analogs in daily clinical practice. Diabetes Res Clin Pract. 2010; 88 Suppl 1:S11-6.

8. Inzucchi SE, Bergenstal RM, Buse JB et al. Management of hyperglycaemia in type 2 diabetes: A patient-centered approach. Position statement of the American Diabetes Association (ADA) and the European Association for the Study of Diabetes (EASD). Diabetologia. 2012; 55(6):1577-96.

9. Home $\mathrm{P}$, Naggar NE, Khamseh $\mathrm{M}$ et al. An observational noninterventional study of people with diabetes beginning or changed to insulin analogue therapy in non-Western countries: the Aichieve study. Diabetes Res Clin Pract. 2011; 94(3):352-63.

10. Philis-Tsimikas A. An update on the use of insulin detemir, with a focus on type 2 diabetes (drug evaluation update). Expert Opin Pharmacother. 2008; 9(12):2181-95.

11. Chapman TM, Perry CM. Insulin detemir: A review of its use in the management of type 1 and 2 diabetes mellitus. Drugs. 2004; 64(22):2577-95.

12. Yang W, Zilov A, Soewondo P, Bech OM, Sekkal F, Home PD. Observational studies: Going beyond the boundaries of randomized controlled trials. Diabetes Res Clin Pract. 2010; 88 Suppl 1:S3-9.

13. Home PD. How can observational trials inform and improve clinical practice? Diabetes Res Clin Pract. 2010; 88 Suppl 1:S1-2.

14. Stratton IM, Adler AI, Neil HA et al. Association of glycaemia with macrovascular and microvascular complications of type 2 diabetes (UKPDS 35): Prospective observational study. BMJ. 2000; 321(7258):405-12. 
15. Gough SC, Tibaldi J. Biphasic insulin aspart in type 2 diabetes mellitus: An evidence-based medicine review. Clin Drug Investig. 2007; 27(5):299-324.

16. Meneghini LF, Rosenberg KH, Koenen C, Merilainen MJ, Lüddeke HJ. Insulin detemir improves glycemic control with less hypoglycemia and no weight gain in patients with type 2 diabetes who were insulin-naive or treated with $\mathrm{NPH}$ or insulin glargine: Clinical practice experience from a German subgroup of the PREDICTIVE study. Diabetes Obes Metab. 2007; 9(3):418-27.

17. Rosenstock J, Davies M, Home PD, Larsen J, Koenen C, Schernthaner G. A randomised, 52-week, treat-to-target trial comparing insulin detemir with insulin glargine when administered as add-on to glucose-lowering drugs in insulin-naive people with type 2 diabetes. Diabetologia. 2008; 51(3):408-16.

18. Swinnen SG, Dain MP, Aronson R et al. A 24-week, randomized, treat-to-target trial comparing initiation of insulin glargine once-daily with insulin detemir twice-daily in patients with type 2 diabetes inadequately controlled on oral glucose-lowering drugs. Diabetes Care. 2010; 33(6):1176-8.

19. Dornhorst A, Lüddeke HJ, Honka M et al. Safety and efficacy of insulin detemir basal-bolus therapy in type 1 diabetes patients: 14week data from the European cohort of the PREDICTIVE study. Curr Med Res Opin. 2008; 24(2):369-76.

20. Sharma SK, Al-Mustafa M, Oh SJ et al. Biphasic insulin aspart 30 treatment in patients with type 2 diabetes poorly controlled on prior diabetes treatment: Results from the PRESENT study. Curr Med Res Opin. 2008; 24(3):645-52.
21. Noh RM, Graveling AJ, Frier BM. Medically minimising the impact of hypoglycemia in type 2 diabetes: A review. Expert Opin Pharmacother. 2011; 12(14):2161-75.

22. UK Hypoglycemia Study Group. Risk of hypoglycemia in types 1 and 2 diabetes: effects of treatment modalities and their duration. Diabetologia. 2007; 50(6):1140-7.

23. Kontodimopoulos N, Pappa E, Chadjiapostolou Z, Arvanitaki E, Papadopoulos AA, Niakas D. Comparing the sensitivity of EQ-5D, SF-6D and 15D utilities to the specific effect of diabetic complications. Eur J Health Econ. 2012; 13(1):111-20.

24. Shah S, Zilov A, Malek R, Soewondo P, Bech O, Litwak L. Improvements in quality of life associated with insulin analogue therapies in people with type 2 diabetes: Results from the Archieve observational study. Diabetes Res Clin Pract. 2011; 94(3):364-70.

25. Dornhorst A, Lüddeke HJ, Koenen $\mathrm{C}$ et al. Transferring to insulin detemir from NPH insulin or insulin glargine in type 2 diabetes patients on basal-only therapy with oral antidiabetic drugs improves glycemic control and reduces weight gain and risk of hypoglycemia: 14-week follow-up data from PREDICTIVE. Diabetes Obes Metab. 2008; 10(1):75-81.

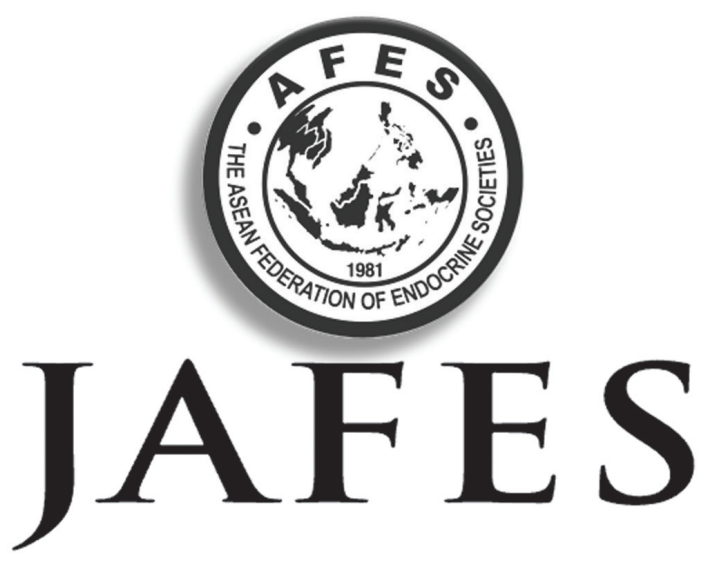

\section{Clinical controversies and disease updates are also welcome. Instructions to Authors available at www.ASEAN-endocrinejournal.org.}

